ARTICLE

Received 22 Apr 2015 | Accepted 16 Jun 2015 | Published 28 Jul 2015

DOI: $10.1038 /$ ncomms8834

OPEN

\title{
Promotion of presynaptic filament assembly by the ensemble of $S$. cerevisiae Rad51 paralogues with Rad52
}

William A. Gaines ${ }^{1, \star}$, Stephen K. Godin ${ }^{2, \star}$, Faiz F. Kabbinavar ${ }^{2}$, Timsi Rao ${ }^{1}$, Andrew P. VanDemark ${ }^{3}$, Patrick Sung ${ }^{1} \&$ Kara A. Bernstein ${ }^{2}$

The conserved budding yeast Rad51 paralogues, including Rad55, Rad57, Csm2 and Psy3 are indispensable for homologous recombination (HR)-mediated chromosome damage repair. Rad55 and Rad57 are associated in a heterodimer, while Csm2 and Psy3 form the Shu complex with Shu1 and Shu2. Here we show that Rad55 bridges an interaction between Csm2 with Rad51 and Rad52 and, using a fully reconstituted system, demonstrate that the Shu complex synergizes with Rad55-Rad57 and Rad52 to promote nucleation of Rad51 on singlestranded DNA pre-occupied by replication protein A (RPA). The csm2-F46A allele is unable to interact with Rad55, ablating the ability of the Shu complex to enhance Rad51 presynaptic filament assembly in vitro and impairing HR in vivo. Our results reveal that Rad55-Rad57, the Shu complex and Rad52 act as a functional ensemble to promote Rad51-filament assembly, which has important implications for understanding the role of the human RAD51 paralogues in Fanconi anaemia and cancer predisposition.

\footnotetext{
${ }^{1}$ Department of Molecular Biochemistry and Biophysics, Yale University School of Medicine, New Haven, Conneticut 06510, USA. ${ }^{2}$ Department of Microbiology and Molecular Genetics, University of Pittsburgh School of Medicine, 5117 Centre Avenue, UPCI Research Pavilion, G5.c, Pittsburgh, Pennsylvania 15217, USA. ${ }^{3}$ Department of Biological Sciences, University of Pittsburgh, Pittsburgh, Pennsylvania 15260, USA. ${ }^{\star}$ These authors contributed equally to this work. Correspondence and requests for materials should be addressed to P.S. (email: patrick.sung@yale.edu) or to K.A.B. (email: karab@pitt.edu).
} 
H omologous recombination (HR) represents a major tool for repairing injured replication forks, chromosomes with DNA double-strand breaks (DSBs), telomere lengthening and ensuring the accurate disjunction of homologous chromosomes in meiosis I (refs 1,2). Defective HR underlies human diseases including cancer and Fanconi anaemia ${ }^{3-7}$. An important conserved HR step is the formation of a Rad51-single-stranded DNA (ssDNA) nucleoprotein filament, also called the presynaptic filament, at DNA damage. In yeast, Rad51 presynaptic filament assembly on ssDNA pre-occupied by RPA, the ubiquitous singlestrand DNA-binding protein, is facilitated by Rad52 (or by BRCA2 in humans) and a heterodimeric complex of the Rad51 paralogues Rad55 and Rad57 (refs 2,8). In conjunction with the DNA motor protein Rad54, the presynaptic filament mediates homology search and strand invasion, and the resultant DNA joint, the D-loop, is resolved by mechanistically distinct pathways to a crossover or non-crossover product.

Recent studies identified the yeast Shu complex as a novel factor that promotes Rad51-dependent repair by an undefined mechanism ${ }^{9-13}$. The Shu complex consists of four subunits that include two Rad51 paralogues (Csm2 and Psy3)Shu1 and Shu2 (ref. 10) and is conserved in humans $9,14,15$. The Shu complex was originally identified during a screen for mutants that suppress the slow growth of top $3 \Delta$ cells and it was shown to act in the RAD52 epistasis group to promote $\mathrm{HR}^{10,12}$. The Shu complex similarly suppresses the HU and MMS sensitivity of $s g s 1 \Delta$ cells, placing it at an early step of $\mathrm{HR}^{10}$. Here we present the first biochemical evidence for a synergistic role of the Shu complex with $\operatorname{Rad} 55-\operatorname{Rad} 57$ and $\operatorname{Rad} 52$ to promote Rad51 presynaptic filament formation on RPA-coated ssDNA. We find by yeast-two-hybrid (Y2H) that Rad55-Rad57 bridges an interaction between the Shu complex and Rad52 and Rad51. In vitro, the Shu complex stimulates Rad51-filament formation in the presence of Rad55-Rad57 and Rad52. Disruption of the Rad55-Csm2 interaction by use of the $\operatorname{csm} 2-F 46 A$ allele blocks this Rad51-filament stimulation in vitro and impairs HR in vivo. Together, our data support a model wherein Csm2 and Rad55 bridge interactions among the Rad55-Rad57 heterodimer, the Shu complex and Rad52 to promote Rad51-dependent homology-directed DNA repair.

\section{Results}

The Shu complex promotes Rad51 presynaptic filament assembly. We first focused on the purified Csm2-Psy3 heterodimer (Supplementary Fig. 1a), as the known protein interaction and DNA-binding activities occur through these subunits, including our previous findings that Csm2 associates with Rad55 in the $\mathrm{Y} 2 \mathrm{H}$ system ${ }^{16}$. Indeed, we found by affinity pull-down that Csm2-Psy3 interacts with Rad55-Rad57 (Fig. 1a and Supplementary Fig. 2a). Similarly, the Shu complex (Csm2-Psy3Shu1-Shu2) interacts with Rad55-Rad57 by pull-down (Supplementary Fig. 2a). We next asked whether Csm2 would interact with Rad52 by $\mathrm{Y} 2 \mathrm{H}$ analysis (Fig. 1b). As shown before, association of Csm2 with Rad55 and self-interaction of Rad52 were seen $^{16-18}$, and, importantly, Csm2 and Rad55 interacted with Rad52 (Fig. 1b). While the Csm2-Rad52 interaction was abolished in rad55 $\Delta$ cells, the Rad52-Rad55 interaction remained without CSM2 (Fig. 1c,d). We employed biochemical pull-down to validate these interactions and found that, consistent with the $\mathrm{Y} 2 \mathrm{H}$ results above, while GST-tagged Rad52 could interact with Rad55-Rad57 (Fig. 1e), its interaction with the Shu complex was negligible. We attempted to validate that Rad55-Rad57 bridges Rad52 to Shu complex by performing the GST pull-down on a mixture of GST-Rad52, Rad55-Rad57 and Shu complex. However, the degree to which Shu complex is pulled down by Rad52 is either not affected or only
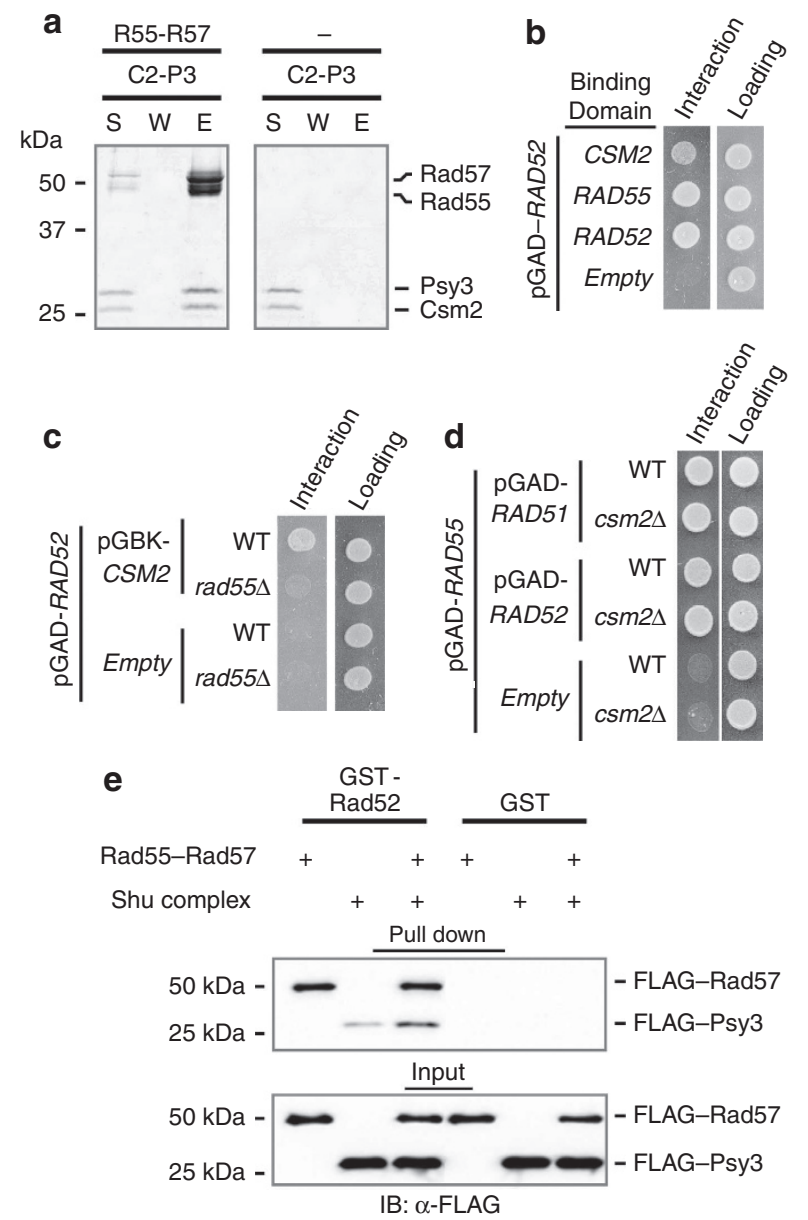

Figure 1 | Interactions of Rad55-Rad57 with the Shu complex and Rad52. (a) Rad55-Rad57 was incubated with Csm2-Psy3 and the protein complex was captured through the (His) ${ }_{6}$ tag on Rad55 using $\mathrm{Ni}^{2+}$ resin. $\mathrm{E}$, eluate from the resin; $\mathrm{S}$, supernatant containing unbound proteins; W, wash of the resin. (b) Rad52 was tested for $\mathrm{Y} 2 \mathrm{H}$ interaction with the indicated proteins. (c) The $\mathrm{Y} 2 \mathrm{H}$ interaction between Rad52 and $\mathrm{Csm} 2$ was examined in wildtype or rad55 $\Delta$ cells. (d) The $\mathrm{Y} 2 \mathrm{H}$ interaction between Rad52 and Rad55 was examined in wild-type or csm2 $\Delta$ cells. (e) GST-tagged Rad52 was mixed with Rad55-Rad57, Shu complex, or both, and protein complexes were captured on glutathione resin. Immunoblotting for the FLAG tag on Rad57 and Psy3 was used to identify Rad55-Rad57 and Shu complex retained on the resin.

modestly affected by the presence of Rad55-Rad57 (Fig. 1e), perhaps because the associations are too labile for the tripartite assembly to persist through washing steps. In agreement with prior $\mathrm{Y} 2 \mathrm{H}$ results ${ }^{16,17}$, pull-down analysis revealed that Rad55-Rad57 interacts with Rad51 while Csm2-Psy3 does so only weakly (Supplementary Fig. 2b). Addition of Csm2-Psy3 does not attenuate Rad55-Rad57 interaction with Rad51, suggesting the interactions are non-competitive. In addition, we have tested RPA in pull-downs and found that it may interact weakly with Rad55-Rad57, though this association could not be validated by Y2H (Supplementary Fig. 2c).

It has been proposed that the Shu complex and Rad55-Rad57 act to promote Rad51 presynaptic filament formation by antagonizing the Srs 2 helicase $\mathrm{e}^{19}$, which negatively regulates HR by disrupting the presynaptic filament ${ }^{20,21}$. Cells lacking Srs2 therefore accumulate ssDNA-bound Rad51. Since Rad54 enhances the ability of the Rad51 presynaptic filament to catalyse 
DNA strand invasion ${ }^{22}$, srs $2 \Delta$ rad54 $\Delta$ double mutants are inviable, likely because they accumulate long-lived presynaptic filaments. Thus, $\operatorname{srs} 2 \Delta$ rad54 $\Delta$ inviability is suppressed by disruption of RAD52, which limits presynaptic filament formation ${ }^{23}$. Similarly, disruption of RAD55 or CSM2 suppresses $s r s 2 \Delta$ rad54A inviability ${ }^{24}$. To expand on these findings, we asked whether disruption of RAD55 or CSM2 suppresses $s r s 2 \Delta$ rad $54 \Delta$ inviability to the same extent as $R A D 52$ deletion by tetrad dissection and spore analysis, and indeed it does (Supplementary Fig. 3, $P$ value $<0.05$ by $\chi^{2}$-test). Thus, the Shu complex and Rad55-Rad57 do not act solely as negative regulators of Srs2 and can function independently of Rad54.

We used several complementary approaches to assess the role of the Shu complex in Rad51 presynaptic filament assembly (Fig. 2a,b, Supplementary Fig. 4). Specifically, we monitored Rad51 loading onto RPA-coated ssDNA bound to magnetic beads (Fig. 2a) and also examined homologous DNA strand exchange activity (Fig. 2b). In the absence of RPA, Rad51 readily gained access to the bead-immobilized ssDNA and efficiently catalysed the exchange of homologous DNA strands (Fig. 2a,b, lane 2). However, RPA on ssDNA strongly attenuated Rad51 loading efficiency and DNA strand exchange (Fig. 2a,b, lane 3). Under the reaction conditions, the Shu complex and Rad55-Rad57 alone or together were insufficient to overcome the inhibitory effect of RPA (Fig. 2a,b, lanes 4-6). The addition of Rad52 permitted Rad51 loading onto RPA-coated ssDNA, but, because of the limiting conditions employed, the amount of ssDNA-associated Rad51 was only a fraction of that achieved when RPA was absent (Fig. 2a,b, lane 7 compared with 2). Rad51 loading in the presence of Rad52 was stimulated by the addition of Rad55-Rad57 but not the Shu complex (Fig. 2a,b, lanes 7 compared with 8 and 9). Importantly, adding Shu complex together with Rad52 and Rad55-Rad57 led to a marked stimulation of Rad51 loading and DNA strand exchange activity (Fig. 2a,b, lane 10). The enhancement of Rad51 loading by Rad55-Rad57 and the Shu complex is most prominent at both 18 and $23^{\circ} \mathrm{C}$ and less pronounced at $30^{\circ} \mathrm{C}$ (Fig. $2 \mathrm{~b}$ and Supplementary Fig. 5a,b). This is consistent with rad55 $\Delta$ cells exhibiting a more severe DNA damage sensitivity at lower temperatures ${ }^{19,25}$. Since we found that Shu complex stimulates Rad51-filament assembly, we next visualized the nucleoprotein products of Rad51 loading using electron microscopy (EM). Consistent with our biochemical data, we observed a significantly greater number of Rad51 filaments in reactions where Rad55-Rad57 and Shu complex were added together with Rad52 (Supplementary Fig. 4). Collectively, these results reveal a synergistic action of Rad52 and the two Rad51 paralogue complexes in enabling utilization of RPA-coated ssDNA for presynaptic filament assembly.

We found that the Csm2-Psy3 heterodimer is just as adept as the full Shu complex in the enhancement of presynaptic filament assembly in the presence of Rad55-Rad57, suggesting that Shu1 and Shu2, which are not Rad51 paralogues, may not be directly involved in presynaptic filament assembly (Supplementary Fig. 6a). The DNA strand exchange reactions performed in Fig. $2 \mathrm{~b}$ contained Rad54 to accelerate the rate of product formation ${ }^{26}$, but similar results were obtained without Rad54 (Supplementary Fig. 6b). Rad55-Rad57 and the Shu complex do not appear to directly enhance the activities of Rad51 or Rad54 since DNA strand exchange reactions lacking RPA are not stimulated by addition of Rad55-Rad57 and Shu complex (Supplementary Fig. 6c).

By X-ray crystallography, several groups have determined the structure of Csm2-Psy3 (refs 27-29). We searched for solvent exposed residues that could participate in interaction with a partner molecule such as Rad55. We applied surface triplet propensity analysis ${ }^{30}$ to this structure ${ }^{29}$ to reveal a potential (i)

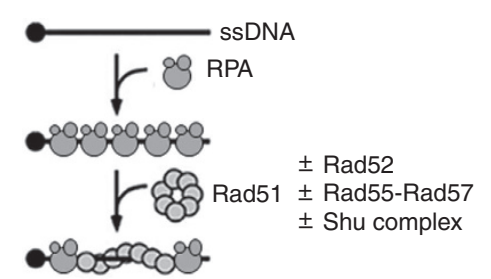

(ii)

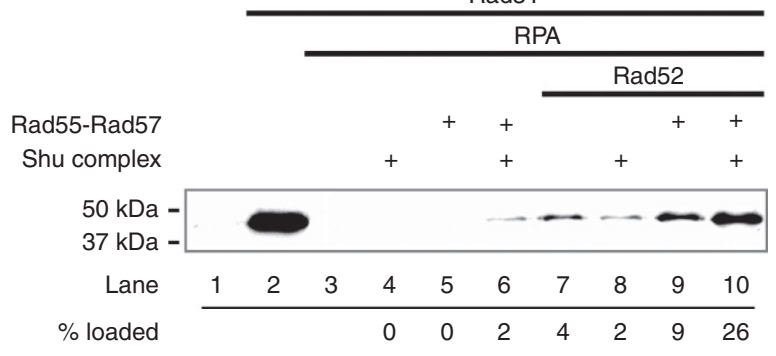

b

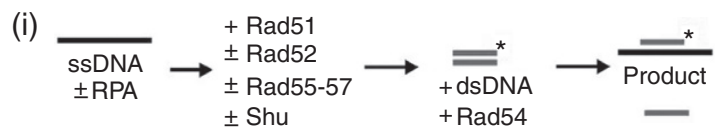

(ii)
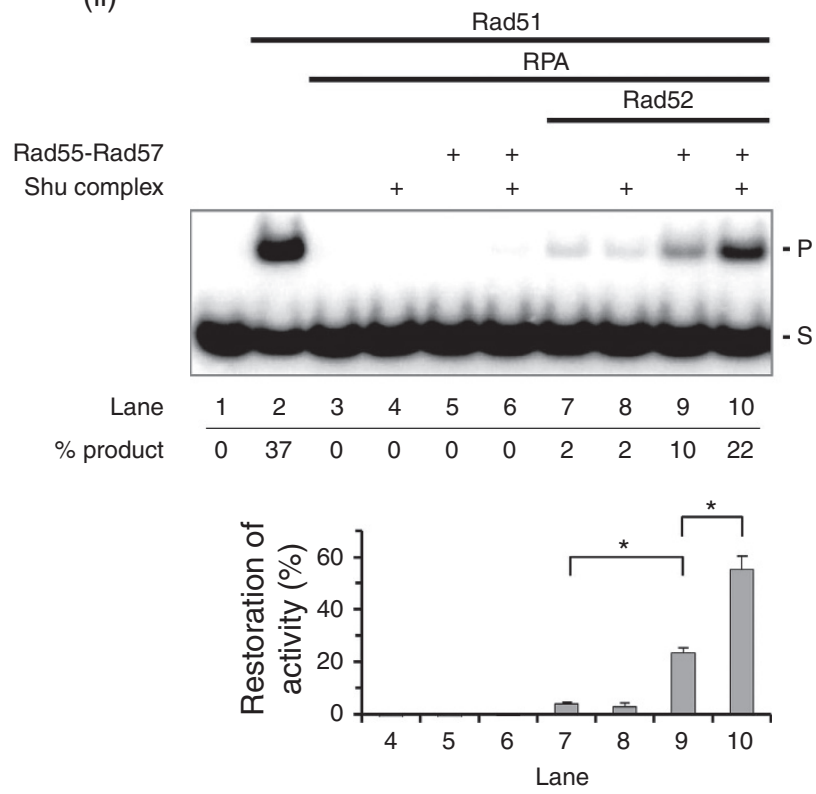

Figure 2 | Enhancement of Rad51 loading onto RPA-coated ssDNA by the Shu complex and Rad55-Rad57. (a) (i) Schematic of the Rad51 loading assay. (ii) Magnetic bead resin with immobilized ssDNA was pre-incubated with RPA, then Rad51 was added along with combinations of Rad52, Shu complex and Rad55-Rad57. The amount of Rad51 retained on the ssDNA was determined by immunoblotting. (b) (i) Schematic of the DNA strand exchange assay. (ii) RPA-coated ssDNA was incubated with Rad51 and combinations of Rad52, Shu complex and Rad55-Rad57, mixed with radiolabelled homologous double-stranded DNA (dsDNA) and Rad54 and then analysed. P, DNA strand exchange product; S, dsDNA substrate. S.d. are plotted as error bars $(n=3)$ and ' ${ }^{* \prime}$ indicates significance.

protein interaction surface on Csm2 (Supplementary Fig. 7a), which is highly conserved in numerous fungal homologues (Supplementary Fig. 7b). The phenylalanine 46 to alanine (F46A) (Fig. 3a and Supplementary Fig. 7) mutation was made, as changing this surface residue is unlikely to affect the stability or folding of Csm2. Importantly, we found that the F46A mutation 
a

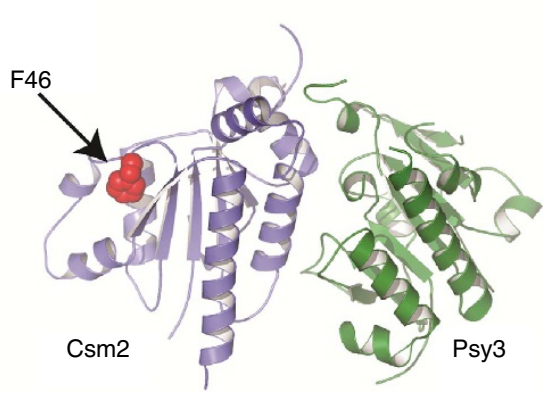

c

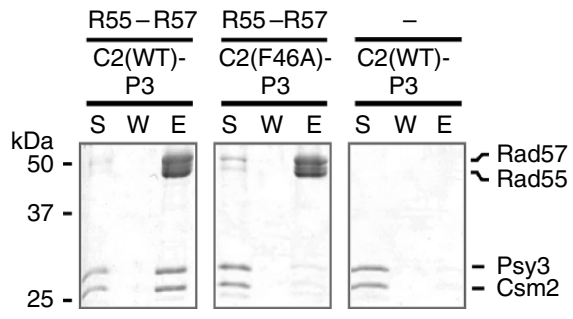

d

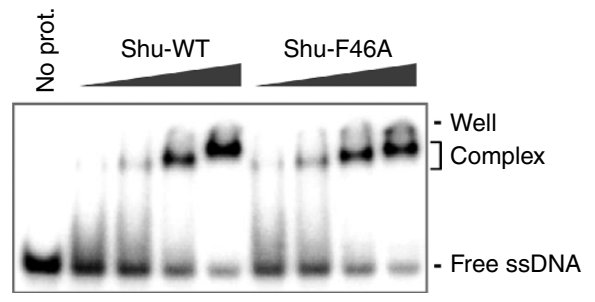

$\mathbf{e}$
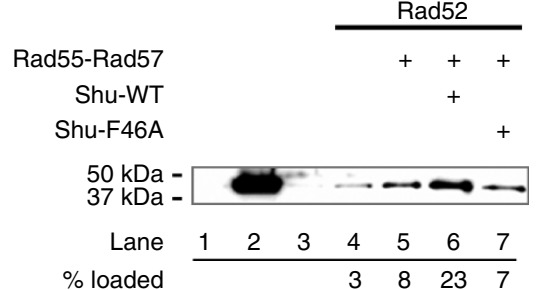

b

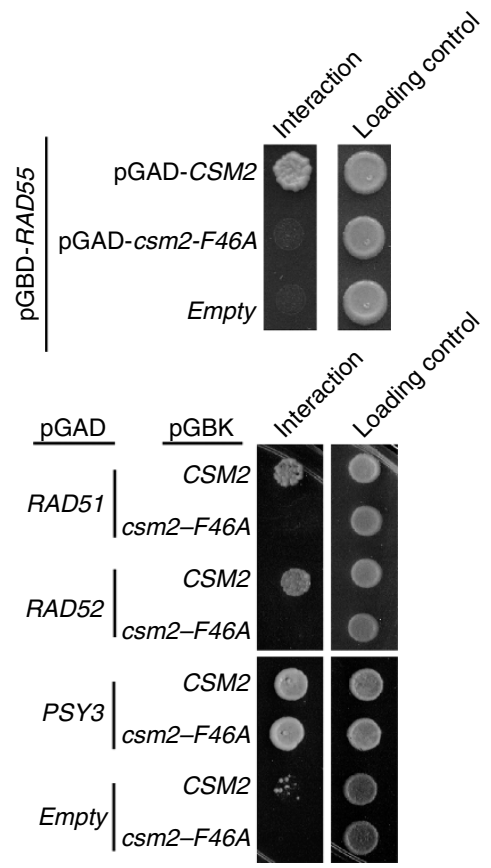

f

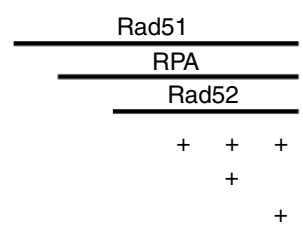

Shu-F46A

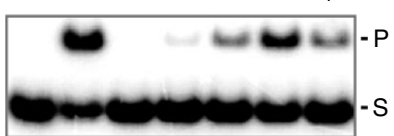

\begin{tabular}{rccccccc} 
Lane & 1 & 2 & 3 & 4 & 5 & 6 & 7 \\
\cline { 2 - 7 }$\%$ product & 0 & 47 & 0 & 2 & 11 & 27 & 14
\end{tabular}

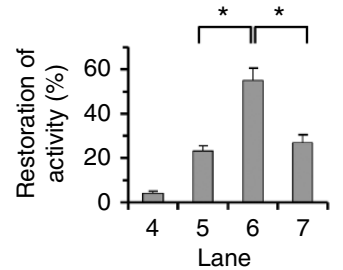

Figure 3 | Impairment of physical interaction and functional synergy of Shu complex with Rad55-Rad57 by the csm2-F46A mutation. (a) Cartoon view of the Csm2-Psy3 heterodimer ${ }^{29}$, with Csm2 in blue and Psy3 in green. Csm2-F46 is highlighted in red. (b) Y2H analysis for interaction of Csm2 and csm2-F46A with Rad55, Rad51, Rad52 and Psy3. (c) Pull-down assay (as in Fig. 1a) to examine interaction of (His) 6 -Rad55-Rad57 with Csm2-Psy3 harbouring csm2-F46A. (d) Analysis of the DNA-binding activity of Shu complex harbouring csm2-F46A. (e) Rad51 loading and (f) DNA strand exchange assays were carried out as in Fig. 2 to evaluate the efficacy of Shu complex harbouring csm2-F46A in the promotion of presynaptic filament assembly. S.d. are plotted as error bars $(n=3)$ and ${ }^{\prime *}$ indicates significance.

disrupts the $\mathrm{Y} 2 \mathrm{H}$ interaction of Csm2 with Rad55 but not Psy3 (Fig. 3b).

We purified Csm2-Psy3 containing the csm2-F46A mutant and verified that it is impaired for Rad55-Rad57 interaction (Fig. 3c) but is proficient in DNA binding (Shu-F46A; Fig. 3d) and complex formation with Shu1-Shu2 (Supplementary Fig. 1). Consistent with Rad55 bridging the interactions between Csm2 with Rad51 and Rad52, we found that the mutant Csm2 does not interact with Rad51 or Rad52 (Fig. 3b), and, importantly, that Shu-F46A is unable to enhance Rad51 presynaptic filament assembly in the bead-based Rad51 loading assay or DNA strand exchange reaction (Fig. 3e,f, compare lane 6 with 7). These results provide the first evidence that the physical interaction between
Csm2 and Rad55-Rad57 is functionally significant and are congruent with our finding that the Shu complex synergizes with Rad55-Rad57 in Rad51 presynaptic filament assembly.

To determine if the Csm2-Rad55 interaction is important for $\mathrm{HR}$, we integrated csm2-F46A into the endogenous CSM2 locus and found that the mutant cells are almost as sensitive to MMS as $\operatorname{csm} 2 \Delta$ cells (Fig. 4a). Furthermore, since previous studies demonstrated that the Shu complex acts upstream of Sgs1, we tested if the csm2-F46A mutation suppresses the MMS and $H U$ sensitivity of an $\operatorname{sgs} 1 \Delta$ mutant like $\operatorname{csm} 2 \Delta$ and found that it does (Fig. 4b). We next employed a direct repeat recombination assay ${ }^{16,31}$ to reveal that $\operatorname{csm} 2-F 46 A$ cells are as impaired as the $\operatorname{csm} 2 \Delta$ mutant in Rad51-mediated gene conversion (Fig. 4c), with 
a

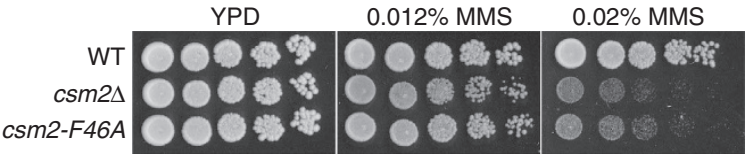

b

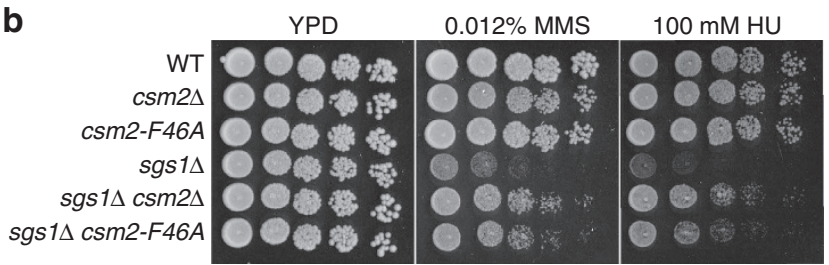

C
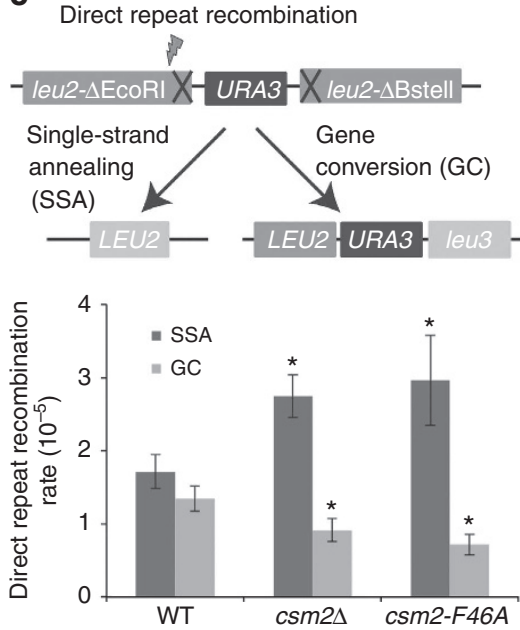

d

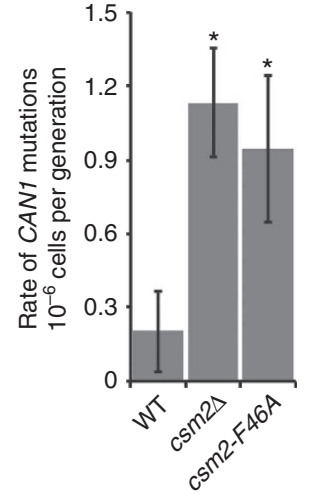

Figure 4 | Impairment of homologous recombination by the csm2-F46A mutation. (a) $c s m 2-F 46 A$ cells are as sensitive to MMS as a $\operatorname{csm} 2 \Delta$ mutant. Cultures were fivefold serially diluted onto YPD medium with the indicated dose of MMS and incubated for 2 days at $30^{\circ} \mathrm{C}$. (b) Similar to $\operatorname{csm} 2 \Delta$, csm2-F46A alleviates the MMS and HU sensitivity of an $\operatorname{sgs} 1 \Delta$ mutant. Cells of the indicated genotypes were fivefold serially diluted and tested for sensitivity to $0.012 \% \mathrm{MMS}$ or $100 \mathrm{mM} \mathrm{HU}$. (c) WT, csm2 $\Delta$ or csm2-F64A cells harbouring a direct repeat HR reporter (leu2- $\triangle \mathrm{EcoRI}:$. URA3::Ileu2$\Delta$ BstEII) were tested for spontaneous rates of Rad51-dependent gene conversion (GC) and Rad51-independent single-strand annealing (SSA) as described ${ }^{16}$. The rates of GC are significantly decreased in both csm2-F46A and $\operatorname{csm} 2 \Delta$ strains $(P<0.01$ by student's $t$-test) with a corresponding increase in SSA relative to WT strains $(P<0.02$ by student's $t$-test). S.d. are plotted as the error bars $(n=3)$ and '*' indicates significance. (d) Like $\operatorname{csm} 2 \Delta$ cells, csm2-F46A cells exhibit an elevated mutation rate in a canavanine mutagenesis assay. S.d. are plotted as error bars $(n=5)$ and ${ }^{\prime}{ }^{\prime \prime}$ indicates significance.

a corresponding increase in Rad51-independent single-strand annealing events (Fig. $4 \mathrm{c}^{16} ; P$ value $<0.02$ by student's $t$-test). Consistent with disruption of other HR factors ${ }^{32}$, using a canavanine mutagenesis assay we found that $\operatorname{csm} 2-F 46 A$ cells accumulate spontaneous mutations like csm2 $($ refs 10,13) (Fig. 4d). Together, our results demonstrate that the interaction between Csm2 and Rad55 is indispensable for the biological efficacy of the Shu complex.

\section{Discussion}

Regulation of Rad51 presynaptic filament formation is essential for HR-mediated repair of damaged DNA and stalled replication forks. Mutations in human mediators of Rad51 presynaptic filament assembly, which is, the hRAD51 paralogues (RAD51B,
RAD51C, RAD51D, XRCC2, XRCC3) or BRCA2, can result in a predisposition to numerous cancers ${ }^{3-7}$. Whether the Rad51 mediators function independently or in a cooperative manner has remained unknown ${ }^{33-35}$. In budding yeast, Rad52 and the Rad55-Rad57 complex have been shown biochemically to promote presynaptic filament formation in isolation. We have furnished evidence that while the Shu complex is devoid of recombination mediator activity, it synergizes with Rad55-Rad57 and Rad52 to mediate the assembly of Rad51 on RPA-coated ssDNA (Supplementary Fig. 8). Our work supports a model in which Rad55-Rad57 bridges an interaction between Rad52 and the Shu complex (Fig. 1) that is functionally important for Rad51 presynaptic filament assembly (Fig. 2, Supplementary Figs 4 and 8). Our study represents the first in vitro reconstitution of Rad51 presynaptic filament assembly being mediated by an ensemble of seven proteins (Rad52, Rad55-Rad57 and Shu1-Shu2-Csm2-Psy3). Furthermore, we have revealed the importance of the Csm2-Rad55 interaction in promoting HR in cells (Fig. 4) and in the facilitation of Rad51 presynaptic filament assembly and homologous DNA pairing and strand exchange in vitro (Fig. 3). Genetic evidence suggests that the Shu complex acts primarily in the repair of stalled replication forks ${ }^{13}$, and while Shul and Shu2 are required for efficient fork repair in vivo they are dispensable for Rad51 loading in vitro (Supplementary Fig. 6a). One possibility is that the Shu complex interaction with Rad55-Rad57 enables its association with replication fork-derived DNA intermediates and subsequently Shul and Shu2 would be necessary for DNA repair progression. Although the function of Shu1-Shu2 remains elusive, previous work suggested that they may regulate Shu complex binding to complex DNA structures produced during $\mathrm{HR}^{29}$, or Shu complex interactions with other HR regulators, such as Srs2 (refs 8,19,36). Intriguingly, the arrangement of the human BCDX2 RAD51 paralogue complex is similar to the Shu complex with Rad55-Rad57 (Supplementary Fig. 9). Given the conservation of the HR machinery, mechanistic insights into the yeast Rad51 paralogues will provide a model for understanding how the human paralogues promote HR. For example, the human RAD51 paralogues may work with BRCA2, the functional equivalent of yeast Rad52, in presynaptic filament assembly ${ }^{37}$. This postulated function of the human RAD51 paralogues is likely important for cancer avoidance ${ }^{3-7}$.

\section{Methods}

Yeast culture methods. The strains used are listed in Supplementary Table 1. Yeast crosses, transformations, tetrad dissections and media preparation were carried out using standard methods ${ }^{16}$. Briefly, yeast diploids were acquired by streaking two haploid yeast strains together on a yeast peptone dextrose (YPD) plate and replica plating onto a double-selective medium that would allow selection for diploids. For tetrad dissections, diploid yeast were plated onto sporulation medium and allowed to sporulate for $72 \mathrm{~h}$ prior to dissection on a Zeiss AX10 dissection scope. Tetrads where grown for 2 days before being genotyped by replica plating onto corresponding selective medium. For plasmid transformations, $50 \mathrm{ml}$ of yeast were grown to mid-log phase $\left(0.3-0.6 \mathrm{OD}_{600}\right)$ in YPD medium, pelleted, resuspended in $100 \mathrm{mM}$ lithium-acetate with $1 \times$ Tris-EDTA solution with $300 \mathrm{ng}$ of plasmid, and then incubated at $30^{\circ} \mathrm{C}$ for $30 \mathrm{~min}$ with $34 \%$ PEG. Cells were heat-shocked at $42{ }^{\circ} \mathrm{C}$ for $15 \mathrm{~min}$ and then plated onto selective medium for each plasmid. The csm2-F46A mutation was introduced into integration, $\mathrm{Y} 2 \mathrm{H}$ and expression vectors by site-directed mutagenesis using primers Csm2.F46A. Forward (5'-GATGCCACAAGCTCAGCTCCGCTAAGTCAATTCC- $\left.3^{\prime}\right)$ and Csm2.F46A. Reverse (5'-GGAATTGACTTAGCGGAGCTGAGCTTGTGGCAT C- $\left.3^{\prime}\right)$. The integration vector was made using primers Csm2.Integration.HindIII (5'-CCCAAGCTTAACAATTCCTCTCTGAGTTGAAA-3') and Csm2. Integration.MfeI ( $5^{\prime}$-CCCCAATTGTATCCTTTAAATAAAACCTGTTTTTCC C- $3^{\prime}$ ) to amplify CSM2 with promoter and terminator from genomic DNA. The amplified DNA was cut with HindIII and MfeI and ligated into yiPLAC211 cut with HindIII and EcoRI.

Y2H analysis. For $\mathrm{Y} 2 \mathrm{H}$ analysis, haploid yeast containing either the $\mathrm{AD}$ or $\mathrm{BD}$ plasmids were mated overnight on YPD, then selected on double-selective media as described above to acquire diploids with both Y2H plasmids (SC-LEU-TRP). For 
Y2H experiments, cells were grown overnight in double-selective liquid medium (SC-LEU-TRP) and then $5 \mu \mathrm{l}$ of yeast at a concentration of $0.5 \mathrm{OD}_{600}$ were plated on selective medium (SC-LEU-TRP-HIS or SC-LEU-TRP) ${ }^{16}$ and incubated at $30^{\circ} \mathrm{C}$ for 2 days.

Serial dilutions. Fivefold serial dilutions were performed with a starting $0.5 \mathrm{OD}_{600}$ using standard techniques ${ }^{16}$. Briefly, yeast were grown overnight, diluted to 0.2 $\mathrm{OD}_{600}$ and incubated at $30^{\circ} \mathrm{C}$ for $3 \mathrm{~h}$ prior to serial dilution.

Mitotic recombination assays. Mitotic recombination assays were performed ${ }^{16,31}$, and recombination rates and standard deviations were calculated using Lea and Coulson's method of the mean ${ }^{38}$. Briefly, for each genotype, nine independent colonies were inoculated into synthetic complete (SC) medium and grown to saturation overnight at $30^{\circ} \mathrm{C}$. Cells were plated onto SC-LEU medium to examine recombination events and SC medium assess cell viability and cell number. After 2 days of growth, the colonies on the SC-LEU plates were replica-plated onto SCURA medium and SC+5-FOA medium to score for Rad51-dependent and independent recombination events, respectively. Each experiment was performed in quadruplicate with s.d. plotted and significance determined by $t$-test.

Canavanine mutagenesis assay. For each trial, individual CAN1 colonies from $\mathrm{WT}, \operatorname{csm} 2 \Delta$ and $\operatorname{csm} 2-F 46 A$ cells were grown in $5 \mathrm{ml} \mathrm{YPD}$ medium overnight at $30^{\circ} \mathrm{C}$, diluted $1: 10$ and $250 \mu \mathrm{l}$ were plated onto SC-ARG + CAN plates. To determine the number of cells plated, the culture was further diluted 1:10,000 and $120 \mu \mathrm{l}$ were plated onto YPD plates. The plates were incubated at $30^{\circ} \mathrm{C}$ for 2 days before colonies were counted. The relative rate of $C A N 1$ inactivation for each strain is reported as an average of five experiments with s.d. plotted.

Suppression of rad54 $\Delta$ srs2 $\Delta$ synthetic lethality. To analyse the suppression of rad $54 \Delta \operatorname{srs} 2 \Delta$ synthetic lethality, a diploid yeast strain heterozygous for the indicated genes was sporulated for $72 \mathrm{~h}$ and tetrad dissected onto rich medium and incubated for 2 days at $30^{\circ} \mathrm{C}$. Three hundred and sixty-one tetrads were genotyped and assessed for viability. The $\chi^{2}$-analysis was used to determine significance for which genotypes were associated with inviability.

\section{Protein expression and purification. Rad51, Rad52, RPA and Rad54 proteins} were prepared as follows ${ }^{39-41}$ :

The Rad55-Rad57 complex was expressed in Saccharomyces cerevisiae strain JEL1 (ref. 42) by using the pESC-URA vector (Agilent) harbouring (His) 6 -tagged RAD55 and FLAG-tagged RAD57 genes; the affinity tags are fused to the N-termini of Rad55 and Rad57 proteins. Cells were grown at $30^{\circ} \mathrm{C}$ until $\mathrm{OD}_{660}$ of 0.7 , then induced for protein expression by the addition of galactose to $2 \%$, cultured for an additional $18 \mathrm{~h}$ and harvested by centrifugation. All the subsequent steps were carried out at $4{ }^{\circ} \mathrm{C}$ in Buffer T $(25 \mathrm{mM}$ Tris- $\mathrm{HCl}, \mathrm{pH} 7.4,10 \%$ glycerol, $0.5 \mathrm{mM}$ EDTA, $0.01 \%$ IGEPAL CA-630 (Sigma), 1 mM DTT) was used throughout protein purification. Importantly, the inclusion of ATP and $\mathrm{Mg}^{2+}$ during lysis (with $5 \mathrm{mM}$ ATP and $2 \mathrm{mM} \mathrm{Mg}^{2+}$ ) and purification steps (with $2 \mathrm{mM}$ of each) helps minimize the aggregation of Rad55-Rad57 and precipitation. To prepare cell lysate, a $40 \mathrm{~g}$ cell pellet was resuspended in $160 \mathrm{ml}$ of buffer with $500 \mathrm{mM} \mathrm{KCl}, 10 \%$ sucrose, $0.1 \%$ IGEPAL and protease inhibitors $(1 \mathrm{mM}$ phenylmethylsulfonyl fluoride and $5 \mu \mathrm{g} \mathrm{ml}^{-1}$ each of aprotinin, chymostatin, leupeptin and pepstatin) then lysed by grinding with dry ice followed by sonication. After ultracentrifugation $(100,000 \mathrm{~g}$ for $1 \mathrm{~h}$ ), the clarified lysate was incubated with $2 \mathrm{ml}$ of $\mathrm{Ni}^{2+}$-NTA affinity resin (Qiagen) for $30 \mathrm{~min}$ with gentle mixing. The resin was poured into a column and washed using buffer with $500 \mathrm{mM} \mathrm{KCl}$ and $20 \mathrm{mM}$ imidazole, followed by elution using wash buffer supplemented with $250 \mathrm{mM}$ imidazole. The eluate was then mixed with $0.5 \mathrm{ml}$ anti-FLAG affinity resin (Sigma) for $2 \mathrm{~h}$ with gentle mixing. The resin was poured into a column and washed using buffer with $200 \mathrm{mM} \mathrm{KCl}$, followed by elution using wash buffer supplemented with $0.3 \mathrm{mg} \mathrm{ml}^{-1}$ FLAG peptide. The protein pool was diluted with an equal volume of buffer $\mathrm{T}$ and fractionated in a $1 \mathrm{ml}$ Mono Q column with a $30 \mathrm{ml}$ gradient of $100-700 \mathrm{mM} \mathrm{KCl}$, collecting $0.5 \mathrm{ml}$ fractions. The peak fractions of Rad55-Rad57 (eluting at $\sim 290 \mathrm{mM} \mathrm{KCl}$ ) were pooled, concentrated in an Amicon Ultra microconcentrator (Millipore) to $0.6 \mathrm{ml}$, and fractionated in a $24 \mathrm{ml}$ SuperDex 200 size exclusion column (GE Healthcare) in buffer with $300 \mathrm{mM} \mathrm{KCl}$, collecting $0.5 \mathrm{ml}$ fractions. The peak fractions of Rad55-Rad57 (eluting at appropriate position for monodisperse, heterodimeric complex) were pooled, concentrated in an Amicon Ultra micro-concentrator to $150 \mu \mathrm{l}$, snap-frozen in liquid nitrogen and stored at $-80^{\circ} \mathrm{C}$. The yield of highly purified Rad55-Rad57 was $\sim 150 \mu \mathrm{g}$

The Shu complex was expressed in Escherichia coli (Rosetta [DE3]) by cotransforming cells with the pET-Duet vector harbouring (His) ${ }_{6}$-tagged Shul and MBP-tagged Shu2 and the pRSF-Duet vector harbouring Strep-tagged Csm2 and FLAG-tagged Psy3. All the affinity tags are fused to the $\mathrm{N}$ terminus of proteins. Cells were grown in $2 \times \mathrm{LB}$ broth supplemented with $0.1 \mathrm{mM} \mathrm{ZnCl}_{2}$ at $37^{\circ} \mathrm{C}$ until $\mathrm{OD}_{600}$ of 0.8 , and protein expression was induced by the addition of IPTG (isopropyl $\beta$-D-1-thiogalactopyranoside) to $0.2 \mathrm{mM}$ and shifted to growth at $16^{\circ} \mathrm{C}$ for $16 \mathrm{~h}$. Cells were harvested by centrifugation. All the subsequent steps were conducted at $4{ }^{\circ} \mathrm{C}$ in buffer $\mathrm{T}$. For lysate preparation, a $40 \mathrm{~g}$ cell pellet was resuspended in $200 \mathrm{ml}$ of buffer with $300 \mathrm{mM} \mathrm{KCl}$ and protease inhibitors as above and then lysed by sonication. After ultracentrifugation $(100,000 \mathrm{~g}$ for $1 \mathrm{~h})$, the clarified lysate was incubated with $2 \mathrm{ml}$ of $\mathrm{Ni}^{2+}$-NTA affinity resin (Qiagen) for 30 min with gentle mixing. The resin was poured into a column and washed using buffer with $150 \mathrm{mM} \mathrm{KCl}$ and $10 \mathrm{mM}$ imidazole, followed by elution using wash buffer supplemented with $200 \mathrm{mM}$ imidazole. The eluate was then mixed with $2 \mathrm{ml}$ amylose affinity resin (NEB) for $2 \mathrm{~h}$ with gentle mixing. The resin was poured into a column and washed with buffer with $150 \mathrm{mM} \mathrm{KCl}$. Tobacco etch virus protease $(10 \mu \mathrm{g})$ was mixed with the resin to cleave at the linker between MBP and Shu2 and thus permit release of the Shu complex from the amylose resin; this incubation was performed overnight at $4{ }^{\circ} \mathrm{C}$. The released Shu complex was mixed with $0.7 \mathrm{ml}$ anti-FLAG affinity resin for $2 \mathrm{~h}$ with gentle mixing. The resin was poured into a column and washed using buffer with $150 \mathrm{mM} \mathrm{KCl}$, followed by elution using wash buffer supplemented with $0.3 \mathrm{mg} \mathrm{ml}^{-1}$ FLAG peptide. The eluate was concentrated in an Amicon Ultra micro-concentrator to $0.6 \mathrm{ml}$ and fractionated in a $24 \mathrm{ml}$ SuperDex 200 column in buffer with $150 \mathrm{mM} \mathrm{KCl}$, collecting $0.5 \mathrm{ml}$ fractions. For both WT and F46A forms, Shu complex eluted at the position for monodisperse tetrameric protein complex. The peak fractions were pooled, concentrated in an Amicon Ultra micro-concentrator to $100 \mu \mathrm{l}$, snap-frozen in liquid nitrogen and stored at $-80^{\circ} \mathrm{C}$. The yield of highly purified Shu complex was $65 \mu \mathrm{g}$.

Affinity pull-down assays. Csm2-Psy3 $(0.5 \mu \mathrm{g})$ was incubated with or without His-tagged Rad55-Rad57 $(0.5 \mu \mathrm{g})$ in $30 \mu \mathrm{l}$ of buffer C $(20 \mathrm{mM}$ MOPS, $\mathrm{pH} 7.2$, $150 \mathrm{mM} \mathrm{KCl}, 10 \%$ glycerol, $0.01 \%$ IGEPAL, $1 \mathrm{mM}$ DTT, $1 \mathrm{mM} \mathrm{ATP,} 2 \mathrm{mM} \mathrm{MgCl}_{2}$, $15 \mathrm{mM}$ imidazole) for $60 \mathrm{~min}$ at $4{ }^{\circ} \mathrm{C}$. The reactions were mixed with $4 \mu \mathrm{l}$ of $\mathrm{Ni}^{2+}$-NTA resin for $30 \mathrm{~min}$ at $4{ }^{\circ} \mathrm{C}$. After washing the resin five times with $150 \mu \mathrm{l}$ of buffer C with $200 \mathrm{mM} \mathrm{KCl}$, bound proteins were eluted with $2 \%$ SDS. The supernatant, elution, and wash fractions were analysed by $15 \%$ SDS-PAGE followed by Coomassie Blue staining. For pull-down via GST-Rad52, proteins were captured on glutathione resin using the same procedure as above but using buffer with $100 \mathrm{mM} \mathrm{KCl}$ and no imidazole, then the various fractions were immunoblotted with $\alpha$-FLAG antibody (Sigma Aldrich, catalogue \#A8592; 1:3,000 dilution) to detect the FLAG-tagged Shu complex and Rad55-Rad57. The input samples shown represent one-thirtieth of the total input. Blot images of the full gel exposure for this experiment are shown in Supplementary Fig. 10a.

Rad51 loading onto ssDNA immobilized on magnetic beads. RPA $(0.4 \mu \mathrm{M})$ was added to biotinylated dT 83-mer ssDNA (1.4 $\mu \mathrm{M}$ nucleotides) coupled to magnetic streptavidin beads (Roche) in $10 \mu$ l of buffer A ( $35 \mathrm{mM}$ MOPS, pH 7.2, $50 \mathrm{mM} \mathrm{KCl}$, $1 \mathrm{mM}$ DTT, $2 \mathrm{mM}$ ATP, $5 \mathrm{mM} \mathrm{MgCl}_{2}, 100 \mu \mathrm{g} \mathrm{ml}^{-1} \mathrm{BSA}$ ) at $37^{\circ} \mathrm{C}$ for $10 \mathrm{~min}$. Excess RPA was removed by magnetic separation. Then, a mixture consisting of the indicated combination of Rad51, Rad52, Rad55-Rad57 and Shu complex was added to the RPA-coated ssDNA resin in $10 \mu \mathrm{l}$ buffer A. The protein concentrations used were $0.75 \mu \mathrm{M} \operatorname{Rad} 51,0.06 \mu \mathrm{M}$ Rad52, $0.11 \mu \mathrm{M}$ Rad55-Rad57 and $0.23 \mu \mathrm{M}$ Shu complex. The reactions were incubated at $18^{\circ} \mathrm{C}$ (except where stated otherwise) for $90 \mathrm{~min}$ with periodic agitation. The beads were briefly washed twice with $10 \mu \mathrm{l}$ buffer A. Proteins were eluted with $2 \%$ SDS and Rad51 amount was determined by immunoblotting with $\alpha$-Rad51 antibody ${ }^{43}$ (1:3,000 dilution). Full blot images for this experiment are shown in Supplementary Fig. 10b.

Homologous DNA pairing and strand exchange reaction. Oligonucleotidebased DNA pairing and strand exchange assay was conducted as described previously $^{44}$ with slight modifications. The 80 -mer ssDNA oligonucleotide $(1.6 \mu \mathrm{M}$ nucleotides) was incubated with RPA $(0.13 \mu \mathrm{M})$ in buffer A at $37^{\circ} \mathrm{C}$ for $10 \mathrm{~min}$. Then, a mixture consisting of the indicated combination of Rad51, Rad52, Rad55Rad57 and Shu complex was incorporated. The protein concentrations used were $0.64 \mu \mathrm{M} \operatorname{Rad} 51,0.05 \mu \mathrm{M}$ Rad52, $0.14 \mu \mathrm{M}$ Rad55-Rad57 and $0.14 \mu \mathrm{M}$ Shu complex. The reactions were incubated at $18^{\circ} \mathrm{C}$ (except where stated otherwise) for $90 \mathrm{~min}$, then spermidine ( $4 \mathrm{mM}$ final concentration) and ${ }^{32} \mathrm{P}$-labeled homologous 40 -mer double-stranded DNA $(0.8 \mu \mathrm{M}$ base pairs final concentration) with Rad54 $(0.035 \mu \mathrm{M}$ final concentration) and an ATP regenerating system $(20 \mathrm{mM}$ creatine phosphate, $30 \mu \mathrm{g} \mathrm{ml}^{-1}$ creatine kinase) were added to complete the reaction (final volume of $12.5 \mu \mathrm{l})$. The reactions were incubated for $30 \mathrm{~min}$ at $18^{\circ} \mathrm{C}$, deproteinized by treatment with $1 \% \mathrm{SDS}$ and $1 \mathrm{mg} \mathrm{ml}^{-1}$ proteinase $\mathrm{K}$ for $5 \mathrm{~min}$ at $37^{\circ} \mathrm{C}$, and then subjected to electrophoresis in a $10 \%$ polyacrylamide gel in TAE buffer ( $40 \mathrm{mM}$ Tris acetate, $\mathrm{pH}$ 7.4, $0.5 \mathrm{mM}$ EDTA). Radiolabelled DNA species were visualized and quantified by phosphorimaging. Please note: a 'no ATP' control could not be performed due to the presence of ATP in the protein storage buffer for Rad55-Rad57.

Electron microscopy. The reactions were set-up similarly to the DNA strand exchange assay for examining Rad51 loading efficiency, except that a longer ssDNA substrate (150-mer) was used and BSA was not included in buffer A. After a $1.5 \mathrm{~h}$ incubation at $18^{\circ} \mathrm{C}$, the reaction was applied to a glow-discharged, carbon-coated EM grid (Ted Pella, Inc.) and stained with $2 \%$ uranyl acetate. The EM images were acquired on an FEI Tecnai T12 transmission electron microscope at magnification of 30,000 with 20 images sampled per grid. About 200 to 400 nucleoprotein particles were counted for each reaction condition. 
DNA-binding assay. The Shu complex $(0.05,0.10,0.20$ and $0.40 \mu \mathrm{M})$ was incubated with radiolabelled 83 -mer ssDNA $(1 \mu$ M nucleotides $)$ in buffer B $(20 \mathrm{mM}$ MOPS, pH 7.2, $1 \mathrm{mM} \mathrm{DTT,} 150 \mathrm{mM} \mathrm{KCl}, 5 \mathrm{mM} \mathrm{MgCl}_{2}$ and $2.5 \mathrm{mM} \mathrm{ATP)} \mathrm{for}$ $10 \mathrm{~min}$ at $30^{\circ} \mathrm{C}$. DNA species were resolved by electrophoresis in an $8 \%$ polyacrylamide gel run in TA buffer ( $40 \mathrm{mM}$ Tris acetate, $\mathrm{pH}$ 7.4) and then visualized and quantified by phosphorimaging.

\section{References}

1. Heyer, W. D. Biochemistry of eukaryotic homologous recombination. Top. Curr. Genet. 17, 95-133 (2007).

2. Symington, L. S., Rothstein, R. \& Lisby, M. Mechanisms and regulation of mitotic recombination in Saccharomyces cerevisiae. Genetics 198, 795-835 (2014).

3. Clague, J. et al. RAD51C germline mutations in breast and ovarian cancer cases from high-risk families. PLoS ONE 6, e25632 (2011).

4. Suwaki, N., Klare, K. \& Tarsounas, M. RAD51 paralogs: roles in DNA damage signalling, recombinational repair and tumorigenesis. Semin. Cell Dev. Biol. 22, 898-905 (2011).

5. Pennington, K. P. \& Swisher, E. M. Hereditary ovarian cancer: beyond the usual suspects. Gynecol. Oncol. 124, 347-353 (2012).

6. Filippini, S. E. \& Vega, A. Breast cancer genes: beyond BRCA1 and BRCA2. Front. Biosci. 18, 1358-1372 (2013)

7. Park, D. J. et al. Rare mutations in XRCC2 increase the risk of breast cancer. Am. J. Hum. Genet. 90, 734-739 (2012).

8. Karpenshif, Y. \& Bernstein, K. A. From yeast to mammals: Recent advances in genetic control of homologous recombination. DNA Rep. (Amst.) 11, 781-788 (2012).

9. Martin, V. et al. Sws1 is a conserved regulator of homologous recombination in

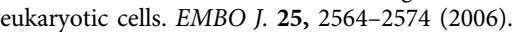

10. Shor, E., Weinstein, J. \& Rothstein, R. A genetic screen for top3 suppressors in Saccharomyces cerevisiae identifies SHU1, SHU2, PSY3 and CSM2: four genes involved in error-free DNA repair. Genetics 169, 1275-1289 (2005).

11. Bernstein, K. A. et al. The Shu complex, which contains Rad51 paralogues, promotes DNA repair through inhibition of the Srs2 anti-recombinase. Mol. Biol. Cell 22, 1599-1607 (2011).

12. Mankouri, H. W., Ngo, H. P. \& Hickson, I. D. Shu proteins promote the formation of homologous recombination intermediates that are processed by Sgs1-Rmi1-Top3. Mol. Biol. Cell 18, 4062-4073 (2007).

13. Ball, L. G., Zhang, K., Cobb, J. A., Boone, C. \& Xiao, W. The yeast Shu complex couples error-free post-replication repair to homologous recombination. Mol. Microbiol. 73, 89-102 (2009).

14. Liu, T., Wan, L., Wu, Y., Chen, J. \& Huang, J. hSWS1.SWSAP1 is an evolutionarily conserved complex required for efficient homologous recombination repair. J. Biol. Chem. 286, 41758-41766 (2011).

15. Godin, S. K. et al. Evolutionary and functional analysis of the invariant SWIM domain in the conserved Shu2/SWS1 protein family from Saccharomyces cerevisiae to Homo sapiens. Genetics 199, 1023-1033 (2015).

16. Godin, S. et al. The Shu complex interacts with Rad51 through the Rad51 paralogues Rad55-Rad57 to mediate error-free recombination. Nucleic Acids Res. 41, 4525-4534 (2013).

17. Xu, X. et al. The yeast Shu complex utilizes homologous recombination machinery for error-free lesion bypass via physical interaction with a Rad51 paralogue. PLoS ONE 8, e81371 (2013).

18. Davis, A. P. \& Symington, L. S. The yeast recombinational repair protein Rad59 interacts with Rad52 and stimulates single-strand annealing. Genetics 159, 515-525 (2001).

19. Liu, J. et al. Rad51 paralogues Rad55-Rad57 balance the antirecombinase Srs2 in Rad51 filament formation. Nature 479, 245-248 (2011).

20. Krejci, L. et al. DNA helicase Srs2 disrupts the Rad51 presynaptic filament. Nature 423, 305-309 (2003).

21. Veaute, X. et al. The Srs2 helicase prevents recombination by disrupting Rad51 nucleoprotein filaments. Nature 423, 309-312 (2003).

22. Petukhova, G., Stratton, S. \& Sung, P. Catalysis of homologous DNA pairing by yeast Rad51 and Rad54 proteins. Nature 393, 91-94 (1998).

23. Esta, A. et al. Rad52 sumoylation prevents the toxicity of unproductive Rad51 filaments independently of the anti-recombinase Srs2. PLoS Genet. 9, e1003833 (2013).

24. St Onge, R. P. et al. Systematic pathway analysis using high-resolution fitness profiling of combinatorial gene deletions. Nat. Genet. 39, 199-206 (2007).

25. Lovett, S. T. \& Mortimer, R. K. Characterization of null mutants of the RAD55 gene of Saccharomyces cerevisiae: effects of temperature, osmotic strength and mating type. Genetics 116, 547-553 (1987).

26. Petukhova, G., Van Komen, S., Vergano, S., Klein, H. \& Sung, P. Yeast Rad54 promotes Rad51-dependent homologous DNA pairing via ATP hydrolysisdriven change in DNA double helix conformation. J. Biol. Chem. 274, 29453-29462 (1999).

27. Sasanuma, H. et al. A new protein complex promoting the assembly of Rad51 filaments. Nat. Commun. 4, 1676 (2013).
28. She, Z. et al. Structural and SAXS analysis of the budding yeast SHU-complex proteins. FEBS Lett. 586, 2306-2312 (2012).

29. Tao, Y. et al. Structural analysis of Shu proteins reveals a DNA binding role essential for resisting damage. J. Biol. Chem. 287, 20231-20239 (2012).

30. Mehio, W., Kemp, G. J., Taylor, P. \& Walkinshaw, M. D. Identification of protein binding surfaces using surface triplet propensities. Bioinformatics 26, 2549-2555 (2010).

31. Smith, J. \& Rothstein, R. An allele of RFA1 suppresses RAD52-dependent double-strand break repair in Saccharomyces cerevisiae. Genetics 151, 447-458 (1999).

32. Huang, M. E., Rio, A. G., Nicolas, A. \& Kolodner, R. D. A genomewide screen in Saccharomyces cerevisiae for genes that suppress the accumulation of mutations. Proc. Natl Acad. Sci. USA 100, 11529-11534 (2003).

33. Gibb, B. et al. Protein dynamics during presynaptic-complex assembly on individual single-stranded DNA molecules. Nat. Struct. Mol. Biol. 21, 893-900 (2014).

34. Shah, P. P. et al. Swi2/Snf2-related translocases prevent accumulation of toxic Rad51 complexes during mitotic growth. Mol. Cell 39, 862-872 (2010).

35. Wright, W. D. \& Heyer, W. D. Rad54 functions as a heteroduplex DNA pump modulated by its DNA substrates and Rad51 during D loop formation. Mol. Cell 53, 420-432 (2014).

36. Ito, T., Chiba, T., Ozawa, R., Yoshida, M. \& Hattori, M. A comprehensive twohybrid analysis to explore the yeast protein interactome. Proc. Natl Acad. Sci. USA 98, 4569-4574 (2001)

37. Jensen, R. B., Ozes, A., Kim, T., Estep, A. \& Kowalczykowski, S. C. BRCA2 is epistatic to the RAD51 paralogs in response to DNA damage. DNA Rep. (Amst.) 12, 306-311 (2013).

38. Lea, D. E. \& Coulson, C. A. The distribution of the numbers of mutants in bacterial populations. J. Genet. 49, 264-285 (1949).

39. Van Komen, S., Macris, M., Sehorn, M. G. \& Sung, P. Purification and assays of Saccharomyces cerevisiae homologous recombination proteins. Methods Enzymol. 408, 445-463 (2006)

40. Raschle, M., Van Komen, S., Chi, P., Ellenberger, T. \& Sung, P. Multiple interactions with the Rad51 recombinase govern the homologous recombination function of Rad54. J. Biol. Chem. 279, 51973-51980 (2004).

41. Song, B. \& Sung, P. Functional interactions among yeast Rad51 recombinase, Rad52 mediator, and replication protein A in DNA strand exchange. J. Biol. Chem. 275, 15895-15904 (2000).

42. Lindsley, J. E. \& Wang, J. C. On the coupling between ATP usage and DNA transport by yeast DNA topoisomerase II. J. Biol. Chem. 268, 8096-8104 (1993).

43. Sugawara, N., Wang, X. \& Haber, J. E. In vivo roles of Rad52, Rad54, and Rad55 proteins in Rad51-mediated recombination. Mol. Cell 12, 209-219 (2003).

44. San Filippo, J. et al. Recombination mediator and Rad51 targeting activities of a human BRCA2 polypeptide. J. Biol. Chem. 281, 11649-11657 (2006).

\section{Acknowledgements}

We thank Rodney Rothstein and Lorraine Symington for providing materials and reagents. This work was supported by the National Institutes of Health grants ES024872, ES015252, ES007061, CA168635, GM088413 and GM101808.

\section{Author contributions}

W.A.G., S.K.G., A.P.V, P.S. and K.A.B. conceived the study. W.A.G., S.K.G., F.F.K. and T.R. conducted the experiments. W.A.G., S.K.G., A.P.V,, P.S. and K.A.B. contributed to the writing of the manuscript.

\section{Additional information}

Supplementary Information accompanies this paper at http://www.nature.com/ naturecommunications

Competing financial interests: The authors declare no competing financial interests

Reprints and permission information is available online at http://npg.nature.com/ reprintsandpermissions/

How to cite this article: Gaines, W. A. et al. Promotion of presynaptic filament assembly by the ensemble of S. cerevisiae Rad51 paralogues with Rad52. Nat. Commun. 6:7834 doi: $10.1038 /$ ncomms8834 (2015)

This work is licensed under a Creative Commons Attribution 4.0 International License. The images or other third party material in this article are included in the article's Creative Commons license, unless indicated otherwise in the credit line; if the material is not included under the Creative Commons license, users will need to obtain permission from the license holder to reproduce the material To view a copy of this license, visit http://creativecommons.org/licenses/by/4.0/ 